\title{
Hyaluronan-Binding Protein Involved in Hyaluronan Depolymerization Is Up-Regulated and Involved in Hyaluronan Degradation in Human Osteoarthritic Cartilage
}

\author{
Hidenori Shimizu, ${ }^{* \dagger}$ Masayuki Shimoda, ${ }^{*}$ Satsuki Mochizuki, ${ }^{*}$ Yuka Miyamae, ${ }^{*}$ Hitoshi Abe, ${ }^{*}$ Miyuki Chijiiwa, \\ Hiroyuki Yoshida, Jun Shiozawa, ${ }^{\S \Phi}$ Muneaki Ishijima, ${ }^{\S \Phi}$ Kazuo Kaneko, ${ }^{\S \Phi}$ Arihiko Kanaji, ${ }^{\dagger}$ Masaya Nakamura, \\ Yoshiaki Toyama, ${ }^{\dagger}$ and Yasunori Okada*ף
}

From the Departments of Pathology* and Orthopaedic Surgery, ${ }^{\dagger}$ Keio University School of Medicine, Tokyo; the Department of Biological Science Research, ${ }^{\ddagger}$ Kao Corporation, Kanagawa; and the Departments of Medicine for Orthopaedics and Motor Organ ${ }^{\S}$ and Pathophysiology for Locomotive and Neoplastic Diseases, "Juntendo University Graduate School of Medicine, Tokyo, Japan

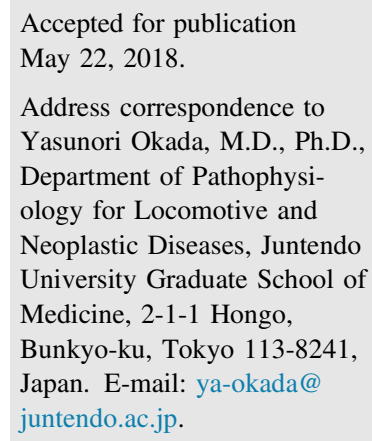

\begin{abstract}
Hyaluronan (HA)-binding protein involved in HA depolymerization (HYBID), also called cell migrationinducing protein (CEMIP; alias KIAA1199), plays a key role in the degradation of HA in skin and arthritic synovial fibroblasts, but its functions in osteoarthritic $(O A)$ cartilage remain elusive. Here, we investigated the expression and roles of HYBID in human OA cartilage. HYBID was highly expressed by chondrocytes in the HA-depleted area of OA cartilage, and HYBID immunoreactivity was correlated with Mankin score, the histopathologic severity of $O A$ lesions of cartilage. Real-time quantitative PCR indicated that HYBID expression was significantly higher in $\mathrm{OA}$ cartilage than in control cartilage. In addition, $O A$ chondrocytes exhibited HA-degrading activity, which was abolished by knock-down of HYBID by siRNAs. Although OA chondrocytes also expressed certain levels of hyaluronidases 1 and 2 and CD44, knock-down of these molecules exhibited negligible effects on HA degradation. Double immunostaining of HYBID and clathrin heavy chain revealed that HYBID was localized in the clathrin-coated vesicles, and $\mathrm{HA}$ was endocytosed within the vesicles of $\mathrm{OA}$ chondrocytes. Among eight factors including cytokines and growth factors examined, only tumor necrosis factor $\alpha$ stimulated $0 \mathrm{~A}$ chondrocytes to overexpress HYBID. These data are the first to demonstrate that HYBID is up-regulated in OA cartilage, and suggest that tumor necrosis factor $\alpha$-stimulated HYBID plays a role in HA degradation in OA cartilage. (Am J Pathol 2018, 188: 2109-2119; https://doi.org/10.1016/j.ajpath.2018.05.012)
\end{abstract}

Osteoarthritis (OA) is one of the most common joint diseases and is characterized by excessive degeneration of extracellular matrix components in the articular cartilage. The extracellular matrix of cartilage is composed mainly of the hyaluronan (HA)-aggrecan (a major cartilage proteoglycan) networks and the collagen fibrils of types II and XI collagens. The initial pathologic change of the articular cartilage in OA patients is the depletion of HA-aggrecan networks, which is followed by the degradation of collagen fibrils. ${ }^{1,2}$ Degradation of fibrillary collagens is ascribed to the proteinase actions of the matrix metalloproteinase family members with collagenolytic activity, such as matrix metalloproteinases 1,13 , and 14, and aggrecan appears to be degraded mainly by a disintegrin and metalloproteinase with thrombospondin motifs 4 and 5, also called aggrecanases 1 and 2 , respectively. ${ }^{2-4}$ Overexpression of these matrix metalloproteinases and a disintegrin and metalloproteinase with thrombospondin motifs 4 and 5 by chondrocytes in OA

Supported by the Japan Society for the Promotion of Science Grants-inaid for Scientific Research 16H05454 (Y.O.) and 16K08719 (M.S.).

H.S. and M.S. contributed equally to this work.

Disclosures: H.Y. is an employee of Kao Corporation and a holder of the HYBID patent. 
cartilage has been reported, ${ }^{5-8}$ and they are suggested to play a central role in collagen and aggrecan degradation in OA cartilage. ${ }^{1,2}$

HA is depolymerized by the action of HA-binding protein involved in HA depolymerization (HYBID) in human skin and synovial fibroblasts independently from hyaluronidases (HYALs) 1 and 2 and CD44. ${ }^{9,10}$ HYBID was originally reported as a deafness gene (KIAA1199) of unknown function, ${ }^{11}$ and HYBID is also known as cell migration-inducing protein (CEMIP). ${ }^{12}$ HYBID is localized in the clathrin-coated vesicles in skin fibroblasts and contributes to depolymerization of high-molecular-weight HA of approximately $10,000 \mathrm{kDa}$ into intermediate-sized HA fragments of 100 to $10 \mathrm{kDa}$, which are released to extracellular milieu. ${ }^{9}$ HYBID is an N-glycosylated protein of $153 \mathrm{kDa}$ composed of one G8, two GG, and four PbH1 domains, lacking substantial homology to HYAL enzymes, HA-binding proteins, HA-link modules, transmembrane domain, and bacterial HYALs. HYBID selectively binds to HA without interaction with chondroitin sulfate A, C, or D; dermatan sulfate; heparin; or heparin sulfate. ' HYBIDmediated degradation of high-molecular-weight HA by synovial fibroblasts may explain the accumulation of lowermolecular-weight HA species in the synovial fluid of patients with joints affected by OA or rheumatoid arthritis. ${ }^{9}$ However, no or little information is available on HYBID expression or its role in HA degradation in OA cartilage. Transforming growth factor (TGF) $\beta_{1}$ down-regulates $H Y B I D$ gene (CEMIP) expression in skin fibroblasts, but the down-regulation by TGF- $\beta_{1}$ is insufficient in OA and rheumatoid arthritis synovial fibroblasts, suggesting that HYBID expression is cell-type specific. ${ }^{10}$ Therefore, if $H Y B I D$ is expressed in OA chondrocytes, regulation of the gene expression by factors such as cytokines and growth factors remains elusive.

Here, we examined the expression and localization of HYBID in human articular cartilage tissues, and found that HYBID is up-regulated by chondrocytes in HA-depleted areas of the OA cartilage and that immunolocalization of HYBID directly correlates with Mankin score. We also provide evidence that HYBID is implicated in the degradation of high-molecular-weight $\mathrm{HA}$ and that among the factors present in OA joints, tumor necrosis factor (TNF)- $\alpha$ up-regulates HYBID expression in OA chondrocytes.

\section{Materials and Methods}

\section{Clinical Samples and Histology}

Nonosteophytic articular cartilage samples were obtained from knee $(n=45)$ and hip $(n=16)$ joints at the time of arthroplasty in patients (mean \pm SD age, $70.8 \pm 11.0$ years) with OA diagnosed according to the criteria of the American College of Rheumatology. ${ }^{13,14}$ Normal (control) cartilage samples were obtained from hip joints $(n=14)$ in patients with femoral neck fracture (mean \pm SD age, $74.5 \pm 11.4$ years). The cartilage tissue samples were fixed with $4 \%$ paraformaldehyde, decalcified with 10\% EDTA ( $\mathrm{pH} 7.4$ ), and embedded in paraffin. Paraffin sections were stained with hematoxylin and eosin, toluidine blue, or Safranin O, and subjected to histologic and histochemical grading described by Mankin et al. ${ }^{15}$ Written informed consent for the experimental use of the surgical samples according to the hospital ethics guidelines was obtained from the patients. The study protocols, which complied with the principles outlined in the Declaration of Helsinki, were approved by the Keio University School of Medicine Ethics Committee (number 20120046) and the Ethics Committee Review Board at Juntendo University (number 15-074).

\section{Immunohistochemistry}

Paraffin sections were subjected to antigen retrieval by boiling in $10 \mathrm{mmol} / \mathrm{L}$ citrate buffer $(\mathrm{pH} 6.0)$ for 10 minutes and immunostaining with rat monoclonal anti-HYBID antibody $^{9}$ or nonimmune control IgG (BD Pharmingen, San Jose, CA). After the reaction with peroxidaseconjugated secondary antibodies (EnVision+; Dako Cytomation, Tokyo, Japan), color was developed with 3,3'diaminobenzidine and sections were counterstained with hematoxylin. ${ }^{16}$ Immunoreactivity of HYBID (the percentage ratio of immunostained chondrocytes to total chondrocytes) in $\mathrm{OA}$ and normal control cartilage was determined by counting the numbers of immunostainpositive chondrocytes and total chondrocytes in the fullthickness area of the cartilage samples. ${ }^{16}$ Mankin scores in the corresponding areas on immunostaining were calculated in the serial sections stained with hematoxylin and eosin or toluidine blue. The relationship between HYBID immunoreactivity and Mankin scores was analyzed by Spearman rank correlation with simple linear regression. ${ }^{16}$

\section{Staining of HA}

The localization of HA was determined using the biotinylated HA-binding protein by modification of the technique described previously. ${ }^{17}$ After blocking endogenous peroxidase and incubating with Proteinase K $(2 \mu \mathrm{g} / \mathrm{mL}$; Dako Cytomation) at $37^{\circ} \mathrm{C}$ for 20 minutes, paraffin sections were incubated with biotinylated HA-binding protein (2 $\mu \mathrm{g} / \mathrm{mL}$; Hokudo, Sapporo, Japan) in phosphate-buffered saline containing $10 \%$ fetal bovine serum. After washing in phosphate-buffered saline, they were reacted with streptavidin-horseradish peroxidase (BD Pharmingen), washed in phosphate-buffered saline, and then incubated in a solution of 3, 3'-diaminobenzidine tetrahydrochloride (Sigma-Aldrich, St. Louis, MO). Specificity of the staining was confirmed by performing the HA staining using biotinylated HA-binding protein on the paraffin sections, which were incubated with Streptomyces HYAL (200 TRU/mL; Seikagaku, Tokyo, Japan), a selective carbohydratedigesting enzyme, in $100 \mathrm{mmol} / \mathrm{L}$ sodium acetate at $60^{\circ} \mathrm{C}$ 
for 2 hours. The sections were counterstained with hematoxylin.

Detection of HYBID, HYAL1, HYAL2, and CD44 Expression by Real-Time Quantitative PCR in Cartilage Samples

$\mathrm{OA}$ and normal cartilage tissues were removed from the articular cartilage with macroscopically definite OA changes or normal appearance, respectively, minced and then freezemilled under liquid nitrogen into a fine powder using CoolMill (Toyobo Life Science, Tokyo, Japan). Total RNA was isolated from the powder and reverse-transcribed to cDNA as described previously. ${ }^{18}$ The expression levels of the $H Y B I D$ gene relative to glyceraldehyde-3-phosphate dehydrogenase gene $(G A P D H)$ were determined by a SYBR Green real-time PCR assay (InvitroGen, Carlsbad, CA) according to the $\Delta \Delta \mathrm{C}_{\mathrm{T}}$ method. ${ }^{18}$ The expression of HYAL1, HYAL2, and CD44 in OA cartilage and normal cartilage was also examined by real-time quantitative PCR. ${ }^{18}$ The primers for HYBID, HYAL1, HYAL2, CD44, and $G A P D H$ were previously described. ${ }^{9}$ The total gene specificity of the sequences of these primers, and the absence of DNA polymorphisms, were ascertained through the use of BLASTN (https://blast.ncbi.nlm.nih.gov/Blast. cgi?PAGE_TYPE = BlastSearch; last accessed January 9, 2018) and Entrez (https://www.ncbi.nlm.nih.gov/Class/ MLACourse/Original8Hour/Entrez; last accessed January $9,2018)$.

\section{Immunoblot Analysis for HYBID}

Proteins were extracted from the powder of OA and normal cartilage by incubation with 10 volumes of $4 \mathrm{~mol} / \mathrm{L}$ guanidine hydrochloride, $10 \mathrm{mmol} / \mathrm{L}$ EDTA, $100 \mathrm{mmol} / \mathrm{L}$ amino caproic acid, and $50 \mathrm{mmol} / \mathrm{L}$ sodium acetate $(\mathrm{pH} 6.8)$ for 48 hours at $4^{\circ} \mathrm{C}$. The samples were dialyzed against 50 $\mathrm{mmol} / \mathrm{L}$ Tris- $\mathrm{HCl}$ buffer $(\mathrm{pH} 7.5)$ containing $5 \mathrm{mmol} / \mathrm{L}$ EDTA and subjected to SDS-PAGE, followed by immunoblot analysis with rat monoclonal anti-HYBID antibody ${ }^{9}$ and anti-GAPDH antibody (Abcam, Cambridge, UK). As a positive control, cell lysates of HEK293 cells transfected with HYBID $\mathrm{cDNA}^{9}$ were subjected to immunoblot analysis for HYBID and GAPDH.

\section{OA Chondrocyte Cultures}

Chondrocytes were isolated from human OA cartilage by enzymatic dissociation and cultured in Dulbecco's modified Eagle's medium/Ham's F-12 (Sigma-Aldrich) supplemented with $10 \%$ fetal bovine serum and $25 \mu \mathrm{g} / \mathrm{mL}$ ascorbic acid as previously described. ${ }^{16}$ They were used for experiments at passage 1 to 2 , and a chondrocytic phenotype of the cultured OA cells was confirmed by the positive immunostaining of aggrecan and type II collagen as previously described. ${ }^{19}$
Transfection of siRNAs for HYBID, HYAL1, HYAL2, and CD44

Two different siRNAs designed to target HYBID, HYAL1, HYAL2, or CD44 and nonsilencing control RNAs were purchased from InvitroGen as described previously. Transfection of OA chondrocytes was performed by electroporation using a Nucleofector kit (Amaxa, Gaithersburg, MD) according to the manufacturer's protocols. Cultured OA chondrocytes (passage 1 to 2) were removed from a dish by enzymatic digestion with collagenase (Roche, Tokyo, Japan) and pronase (Merck, Darmstadt, Germany), divided into three groups, and transfected with nonsilencing RNA, siRNA1, or siRNA2. The transfected chondrocytes used in the comparative studies were always at the same passage number. The expression levels of HYBID and CD44 were detected by immunoblot analysis with anti-HYBID antibody $^{9}$ and anti-CD44 antibody (Santa Cruz Biotechnology, Santa Cruz, CA), respectively, and those of HYALl and HYAL2 were examined by RT-PCR, ${ }^{9}$ because antibodies specific to these HYALs were not available.

\section{Assay for Cellular $\left[{ }^{3} \mathrm{H}\right] \mathrm{HA}$ Depolymerization}

High-molecular-weight $\left[{ }^{3} \mathrm{H}\right]$-labeled $\mathrm{HA} \quad\left(\left[{ }^{3} \mathrm{H}\right] \mathrm{HA}\right)$ of $>1000 \mathrm{kDa}$ was prepared as described previously. ${ }^{9}$ Cellular HA depolymerization was assayed by culturing confluent cells in medium containing $\left[{ }^{3} \mathrm{H}\right] \mathrm{HA}(40,000 \mathrm{dpm} / \mathrm{mL})$ and applying the media to a Sepharose CL-2B column $(1 \times 60$ $\mathrm{cm}$; GE Healthcare, Tokyo, Japan) equilibrated with 0.5 $\mathrm{mol} / \mathrm{L} \mathrm{NaCl}$ in distilled water. The radioactivity of each fraction was measured by a scintillation counter (LSC-6100; Aloka, Tokyo, Japan). The column was calibrated with the fluoresceinamine-labeled HA species: H1 $(1760 \mathrm{kDa}$; peak top kDa), M1 (907 kDa), L1 (197 kDa), S1 (56 kDa), T1 (28 $\mathrm{kDa}$ ), and U1 (9.8 kDa) (PG Research, Tokyo, Japan). An excitation wavelength of $490 \mathrm{~nm}$ and an emission wavelength of $525 \mathrm{~nm}$ were used for the detection of fluoresceinamine.

Immunofluorescence Microscopy for HYBID and Clathrin Heavy Chain and Cellular Distribution of Exogenously Added HA in OA Chondrocytes

OA chondrocytes grown on the Lab-Tek chamber slides (Nalge Nunc International, Rochester, NY) at $70 \%$ to $80 \%$ confluence were fixed with $4 \%$ paraformaldehyde, permeabilized by incubating in phosphate-buffered saline containing $0.05 \%$ Tween 20, and double-immunostained with rat anti-HYBID antibody conjugated to Alexa Flour 488 and goat anti-clathrin heavy chain antibody conjugated to Alexa Flour 555. ${ }^{9}$ The cells were counterstained with TO-PRO-3 (InvitroGen) and mounted in Vectashield (Vector Laboratories, Burlingame, CA). As for controls, chondrocytes were reacted with nonimmune IgG conjugated to Alexa Flours. Conjugation of Alexa Flours to antibodies 
and nonimmune $\operatorname{IgG}$ was performed by Alexa Fluor antibody labeling kit (InvitroGen) according to the manufacturer's protocol. For the detection of exogenously added $\mathrm{HA}$, OA chondrocytes on chamber slides were incubated in the presence or absence of $0.1 \mathrm{mg} / \mathrm{mL}$ biotin-labeled highmolecular-weight HA of $1410 \mathrm{kDa}$ (PG Research) at $37^{\circ} \mathrm{C}$ for 1 hour. After fixation with $4 \%$ paraformaldehyde and permeabilization in phosphate-buffered saline containing Tween 20, they were incubated with streptavidin conjugated to Alexa Fluor 555, counterstained with TO-PRO-3, and mounted in Vectashield. For controls, the cells were incubated with biotin-labeled high-molecular-weight HA digested with Streptomyces HYAL (Seikagaku), followed by the immunostaining described in the previous paragraph. These samples were observed by confocal microscope (LSM 510; Carl Zeiss, Jena, Germany).

\section{Stimulation of OA Chondrocytes with Factors Including Cytokines and Growth Factors}

Serum-starved OA chondrocytes were treated with TNF- $\alpha$ (Dainippon Sumitomo Pharma, Tokyo, Japan), TGF- $\beta_{1}$ (R\&D Systems, Minneapolis, MN), IL-1 $\alpha$ (Dainippon Sumitomo Pharma), histamine (Wako, Osaka, Japan), insulin-like growth factor 1 (Sigma-Aldrich), vascular endothelial growth factor 165 (R\&D Systems), basic fibroblast growth factor (Sigma-Aldrich), prostaglandin $\mathrm{E}_{2}$ (Sigma-Aldrich), or vehicle alone in serum-free Dulbecco's modified Eagle's medium/F-12 containing 0.2\% lactalbumin hydrolysate for 24 hours. The expression of HYBID under treatment with these factors was analyzed by RT-PCR and a SYBR Green real-time PCR assay. ${ }^{18}$ OA chondrocytes treated with TNF- $\alpha(0,1$, or $10 \mathrm{ng} / \mathrm{mL})$ were also subjected to immunoblot analysis with anti-HYBID antibody, ${ }^{9}$ and degradation of exogenously added high-molecular-weight $\left[{ }^{3} \mathrm{H}\right] \mathrm{HA}$ to chondrocytes cultured under treatment with TNF- $\alpha$ was also examined by gel filtration using a Sepharose CL-2B column (GE Healthcare).

\section{Statistical Analysis}

The $U$-test was used to compare the data from OA and normal cartilage samples. For the data from experiments using OA chondrocytes, statistical significance was determined by a $t$-test. $P$ values of $<0.05$ were considered significant.

\section{Results}

\section{Immunolocalization of HYBID in Articular Cartilage}

OA and normal cartilage samples were subjected to histology and immunohistochemistry using anti-HYBID antibody. Histology of the OA cartilage was various, ranging from surface irregularities to marked fibrillation and fissuring. The distribution of Mankin scores (histologic severity of OA) ranged from 3 to 11 , with a mean \pm SD of $5.36 \pm 2.83$, whereas normal articular cartilage had little or no microscopic changes (Mankin scores 0 to 2; $0.62 \pm 0.66$ ). Immunohistochemical staining showed that HYBID was localized to the cytoplasm of the chondrocytes in OA samples, whereas negligible staining was present in normal samples (Figure 1A). In cartilage with mild to moderate OA severity, HYBID signals were restricted to chondrocytes in the superficial and transitional zones, and chondrocytes in the deeper zones showed negligible immunoreactivity. Cartilage samples with severe OA changes showed the HYBID expression in many chondrocytes, including those located in the transitional and radial zones (Figure 1A). Clustered chondrocytes were also
A
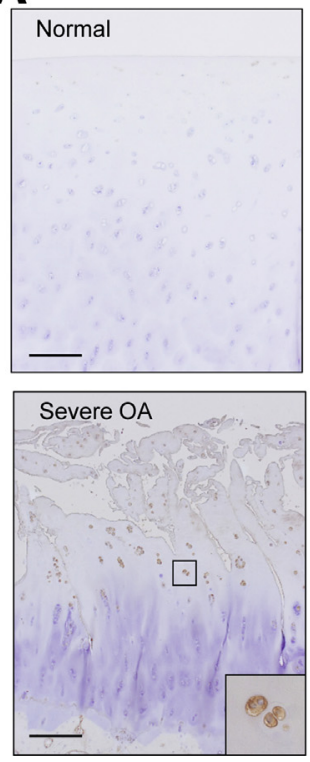

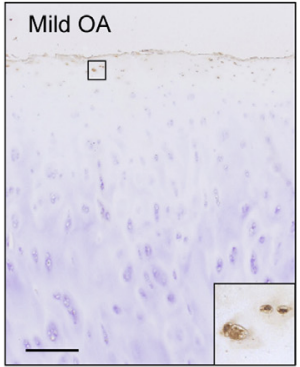

Severe OA (NI)
B

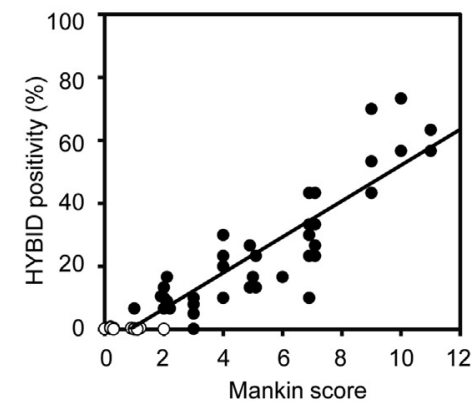

Figure 1 Expression of hyaluronan (HA)-binding protein involved in $\mathrm{HA}$ depolymerization (HYBID) in normal and osteoarthritic (OA) cartilage on immunohistochemistry (IHC) analysis, and correlation between HYBID immunoreactivity and cartilage parameters. A: IHC analysis of HYBID in normal and $\mathrm{OA}$ cartilage samples. Paraffin sections of normal cartilage (Mankin score 1), mild $\mathrm{OA}$ cartilage (Mankin score 4), and severe OA cartilage (Mankin score 8) were immunostained with antiHYBID antibody. Insets show boxed areas at higher magnification. The lower right panel shows severe- $0 \mathrm{~A}$ cartilage immunostained with nonimmune IgG (NI). B: Correlations of HYBID immunoreactivity and Mankin score. Open and closed circles indicate normal $(n=13)$ and OA $(n=39)$ cartilage samples, respectively. Diagonal line shows the estimated regression line superimposed on the scatter diagram. Scale bars $=200 \mu \mathrm{m}$. 
immunohistochemically positive. No immunostaining was observed with nonimmune rat IgG (Figure 1A). Importantly, a strong linear correlation was observed by plotting the HYBID immunoreactivity (the percentage of immunostained chondrocytes to total cells) against Mankin score ( $r=0.91, P<0.0001, n=52$ ) (Figure 1B).

When normal and OA cartilage tissues were stained with Safranin O or HA-binding protein, the cartilage with severe OA changes showed depletion of staining in both the transitional and radial zones in a similar pattern, whereas disappearance of the staining was seen only in the superficial zone in the normal cartilage (Figure 2). Interestingly, HYBID-immunostained OA chondrocytes were located in the areas that were negatively stained by Safranin O or HAbinding protein staining. These data suggest that HYBID expressed by chondrocytes may be involved in HA degradation in OA cartilage.

\section{Expression of HYBID mRNA and Protein in Articular Cartilage}

The relative mRNA expression level of HYBID (HYBID/ $G A P D H$ ratio) was measured by real-time quantitative PCR. The level was approximately 4-fold higher in OA cartilage than in normal control cartilage $(4.14 \pm 4.00$ versus $1.00 \pm 1.42 ; P<0.001$ ) (Figure $3 \mathrm{~A}$ ). Immunoblot analysis of tissue extracts of OA cartilage showed a $150-\mathrm{kDa}$ protein band of HYBID, whereas normal cartilage showed a negligible band (Figure 3B). Since previous studies showed that human chondrocytes express HYAL1, HYAL2, and CD44, ${ }^{20-22}$ the mRNA expression levels of these molecules were examined by real-time quantitative PCR, and it was found that the relative expression levels of HYAL1, HYAL2, and CD44 were also significantly higher in OA cartilage than in normal control cartilage (Figure 3C).

\section{Involvement of HYBID in HA Degradation by $\mathrm{OA}$ Chondrocytes}

To study which molecules are involved in HA degradation in OA chondrocytes, the expression of HYBID, HYAL1, HYAL2, or CD44 was knocked down by siRNAs in OA chondrocytes, and changes in HA-degrading activity were detected by size-exclusion chromatography of highmolecular-weight $\mathrm{HA}$ added to culture media of OA chondrocytes. When OA chondrocytes were transfected with two different siRNAs targeting HYBID (HYBID-1 and $H Y B I D-2$ siRNAs) or nonsilencing control RNA (control siRNA), the expression of HYBID protein was effectively suppressed by $H Y B I D$ siRNAs to $<5 \%$ of the control levels and knockdown of $H Y B I D$ reduced

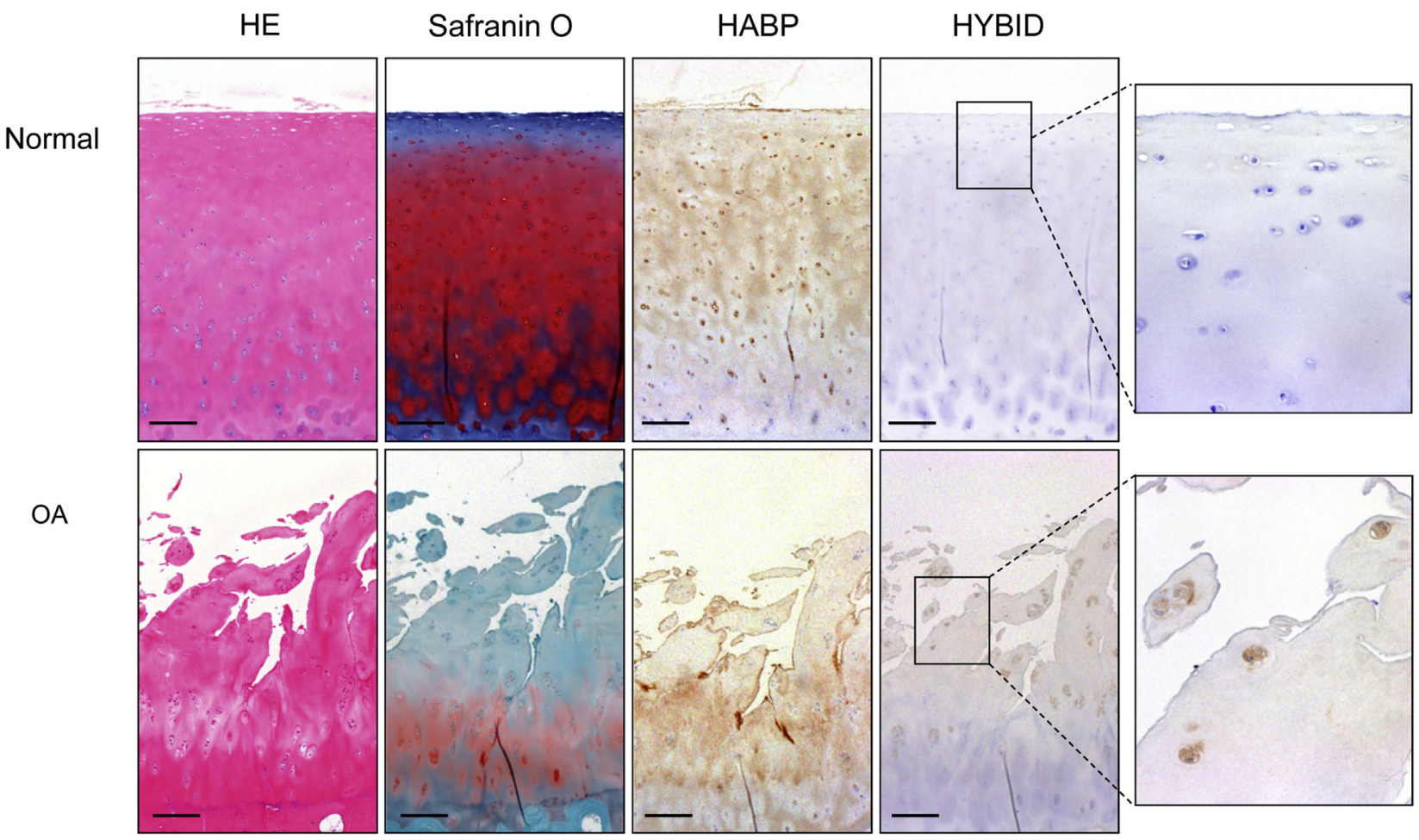

Figure 2 Localization of chondrocytes expressing hyaluronan (HA)-binding protein involved in HA depolymerization (HYBID) in the HA-proteoglycan-depleted area in osteoarthritic $(\mathrm{OA})$ cartilage. Serial sections of normal and $O A$ cartilage samples were subjected to Safranin 0 and HA-binding protein (HABP) staining or immunostaining with anti-HYBID antibody. HYBID-immunostained chondrocytes are located in the Safranin 0- and HABP-negative area of OA cartilage. The boxed areas of HYBID-immunostained sections are shown at higher magnification to the right. Scale bars $=200 \mu \mathrm{m}$. HE, hematoxylin and eosin. 
A

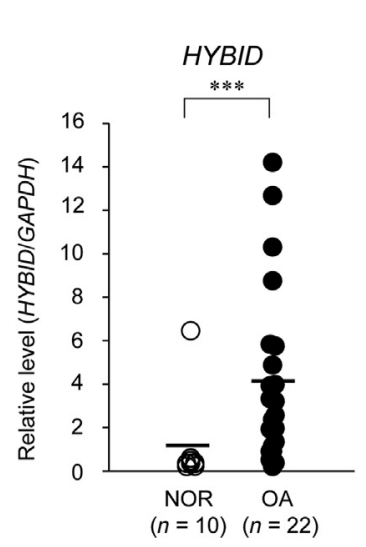

C

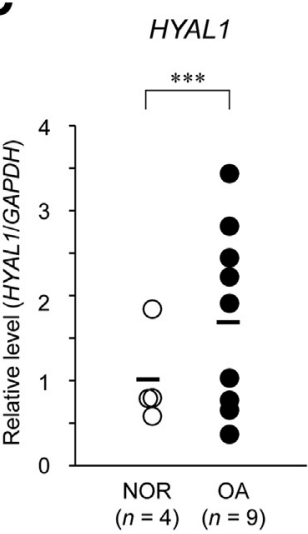

B

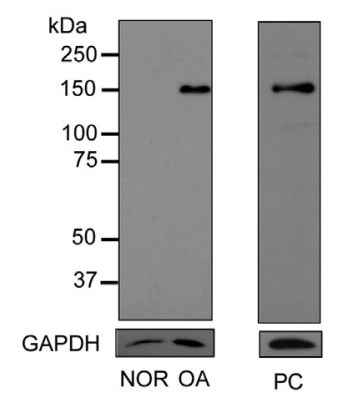

Figure 3 Expression of hyaluronan (HA)-binding protein involved in HA depolymerization (HYBID), HYAL1, HYAL2, and CD44 in normal and osteoarthritic (OA) cartilage. A: Real-time quantitative PCR (qPCR) analysis of HYBID gene expression in normal (NOR) and OA cartilage samples. Relative expression of mRNA for HYBID was determined by normalizing to the expression level of GAPDH transcripts using the $\triangle \Delta C_{\mathrm{T}}$ method. The mean $H Y B I D: G A P D H$ ratio in normal samples is set at 1 . Circles represent individual subjects; horizontal lines indicate means. B: Immunoblot analysis of HYBID in the tissue extracts of NOR and OA cartilage. Proteins were extracted from cartilage tissues, and samples (100 $\mu \mathrm{g} / \mathrm{lane})$ were subjected to sodium dodecyl sulfate-polyacrylamide gel electrophoresis and immunoblot analysis with anti-HYBID antibody. Glyceraldehyde-3-phosphate dehydrogenase (GAPDH) serves as a loading control. C: $q P C R$ analysis of HYAL1, HYAL2, and CD44 gene expression in NOR and $O A$ cartilage samples. Relative expression levels were measured by qPCR as depicted earlier. Circles represent individual subjects; horizontal lines indicate means (A and $\mathbf{C}) .{ }^{* *} P<0.001$. PC, positive control of cell lysate of HEK293 cells transfected with HYBID CDNA. the HA-degrading activity to almost negligible levels (Figure 4A). On the other hand, knockdown of HYAL2 decreased the activity to approximately $50 \%$ of the original (Figure 4C), but no or negligible changes were obtained by knocking down HYAL1 or CD44 (Figure 4, B and D).

\section{Cellular Localization of HYBID and Exogenously Added $\mathrm{HA}$ in OA Chondrocytes}

When immunohistochemistry for HYBID was performed in permeabilized OA chondrocytes, its localization was observed mainly in vesicles at the periphery of the chondrocytes, and no staining was obtained with nonimmune IgG (data not shown). Since HYBID is localized within the clathrin-coated vesicles in skin fibroblasts, double immunostaining of HYBID and the clathrin heavy chain was performed in OA chondrocytes. Signals of both molecules were observed in a vesicular pattern, and HYBID was co-localized with clathrin heavy chain in some vesicles (Figure 5A). In addition, when highmolecular-weight HA was added to the culture media of OA chondrocytes, HA was localized to vesicles at the periphery of these cells, but no fluorescence was shown by incubation with Streptomyces HYAL-digested HA (Figure 5B).
Effects of Factors Including Cytokines and Growth Factors on HYBID Expression in OA Chondrocytes

Serum-starved OA chondrocytes were treated with TNF- $\alpha$, TGF- $\beta_{1}$, IL- $1 \alpha$, histamine, insulin-like growth factor 1 , vascular endothelial growth factor 165 , basic fibroblast growth factor, or prostaglandin $\mathrm{E}_{2}$, and $H Y B I D$ expression was first determined by RT-PCR. Among these factors, the mRNA expression of HYBID appeared to be up-regulated by TNF- $\alpha$, but no definite up- or down-regulation of HYBID was observed by the factors examined (Figure 6A). Real-time quantitative PCR analysis demonstrated the up-regulation of $H Y B I D$ mRNA by TNF- $\alpha$, whereas no significant changes were obtained with other factors used (Figure 6B). The enhanced expression of HYBID by TNF- $\alpha$ was confirmed by densitometric analysis of the protein band of HYBID detected by immunoblot analysis (Figure 6C). In addition, HA-degrading activity was increased by the treatment of OA chondrocytes with TNF- $\alpha$ (Figure 6D), supporting the data of the TNF- $\alpha$-mediated the upregulation of HYBID expression.

\section{Discussion}

In the present study, to the best of our knowledge, we have provided the first evidence that HYBID is overexpressed by 
A

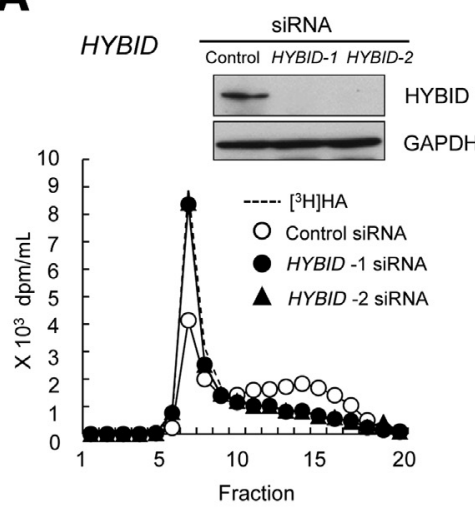

C

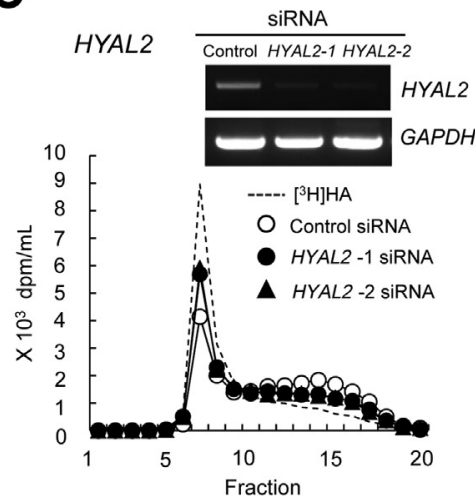

B

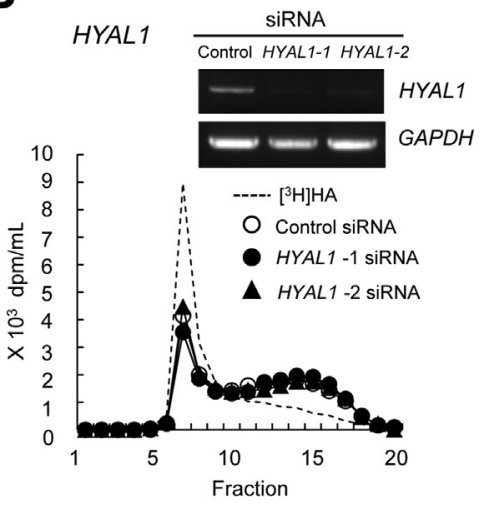

D

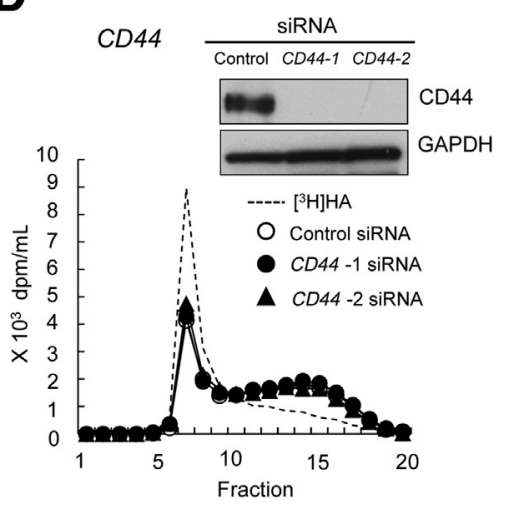

Figure 4 Hyaluronan (HA) degradation mediated by HAbinding protein involved in HA depolymerization (HYBID) in osteoarthritic (OA) chondrocytes. A: Abrogation of HAdegrading activity by knockdown of HYBID with two different siRNAs specific to HYBID. OA chondrocytes transfected with siRNAs for HYBID or nonsilencing RNA (control) were cultured with $\left[{ }^{3} \mathrm{H}\right] \mathrm{HA}$ for 48 hours, and $\mathrm{HA}$-degrading activity was determined by size-exclusion chromatography. Immunoblot analysis for HYBID and glyceraldehyde-3phosphate dehydrogenase (GAPDH) (a loading control) is also shown. B-D: HA-degrading activity in OA chondrocytes, in which HYAL1, HYAL2, or CD44 was knocked down with two different siRNAs for each gene. OA chondrocytes transfected with siRNAs for each gene or nonsilencing RNA (control) were treated with $\left[{ }^{3} \mathrm{H}\right] \mathrm{HA}$, and HA-degrading activity was determined as described earlier. RT-PCR for HYAL1 (B) and HYAL2 (C) and immunoblot analysis for CD44 (D) are also shown. Dashed lines indicate chromatograph for $\left[{ }^{3} \mathrm{H}\right] \mathrm{HA}$ without incubation with chondrocytes; solid lines indicate chromatograph for $\left[{ }^{3} \mathrm{H}\right] \mathrm{HA}$ incubated with chondrocytes transfected with siRNAs. chondrocytes in OA articular cartilage. Histologic, histochemical, and immunohistochemical studies demonstrated that chondrocytes located in the HA-proteoglycan-depleted areas of OA cartilage express HYBID protein, and that the degree of HYBID immunostaining-positive chondrocytes directly correlates with cartilage destruction.

Many organs including the brain, skin, lung, testis, and ovary, but not the liver, are known to express the HYBID gene in humans, ${ }^{23}$ but no information on the expression in articular cartilage was available. In a recent study in HYBID-knockout and wild-type mice, an expression pattern of mouse HYBID similar to that in humans was confirmed, and it was demonstrated that hypertrophic chondrocytes express HYBID during development in wild-type mice, resulting in shorter long bones in the knockout mice. ${ }^{24}$ The phenotype was characterized by suppression of the endochondral ossification, which was caused by the inhibition of vascular endothelial growth factor-mediated angiogenesis and osteoclast recruitment by accumulated high-molecularweight HA in the hypertrophic chondrocyte zone. ${ }^{24}$ Chondrocytes in OA articular cartilage are reported to share the characteristics of hypertrophic chondrocytes by expressing hypertrophic chondrocyte markers such as vascular endothelial growth factor and type $\mathrm{X}$ collagen. ${ }^{25}$ Accelerated turnover of extracellular matrix components, including proteoglycans and HA, is commonly observed both in the hypertrophic chondrocyte zone of the developing growth plate $^{26,27}$ and in OA articular cartilage. ${ }^{1,2}$ Therefore, the finding of HYBID expression by OA chondrocytes located in the HA-deleted area suggests the possible involvement of HYBID in HA degradation in OA cartilage.

Two HYALs (1 and 2) and HA receptor CD44 were originally thought to be crucial to HA degradation through the following steps: high-molecular-weight HA is captured by CD44 on cell surfaces, concentrated in the caveolin-rich lipid rafts, and cleaved by HYAL2 into intermediate-size fragments, which are then transported to lysosomes and finally digested into oligosaccharides by lysosomal enzyme HYAL1 ${ }^{28,29}$ However, since this model was proposed based on the data of the experiments using a breast carcinoma cell $\operatorname{line}^{30}$ and cells stably transfected with these genes, ${ }^{31}$ and because neither HYAL1 nor HYAL2 is expressed in the brain that contains a large amount of HA, ${ }^{32,33}$ the HYAL2/CD44 and HYAL1 system was known to have limitations for the explanation of the rapid catabolism of HA in vivo. ${ }^{9}$ Thus, pathways different from this system were expected to be involved in HA degradation within tissues such as the brain. New HA-degrading pathways were explored and it was verified that HYBID plays a central role in HA degradation independently from HYAL1 and HYAL2/CD44 axis in skin and synovial fibroblasts. ${ }^{9}$ In the present study, HYAL1, HYAL2, and CD44 were also up-regulated in OA cartilage tissue, and knockdown of HYAL2 partially decreased HA-degrading activity in OA 


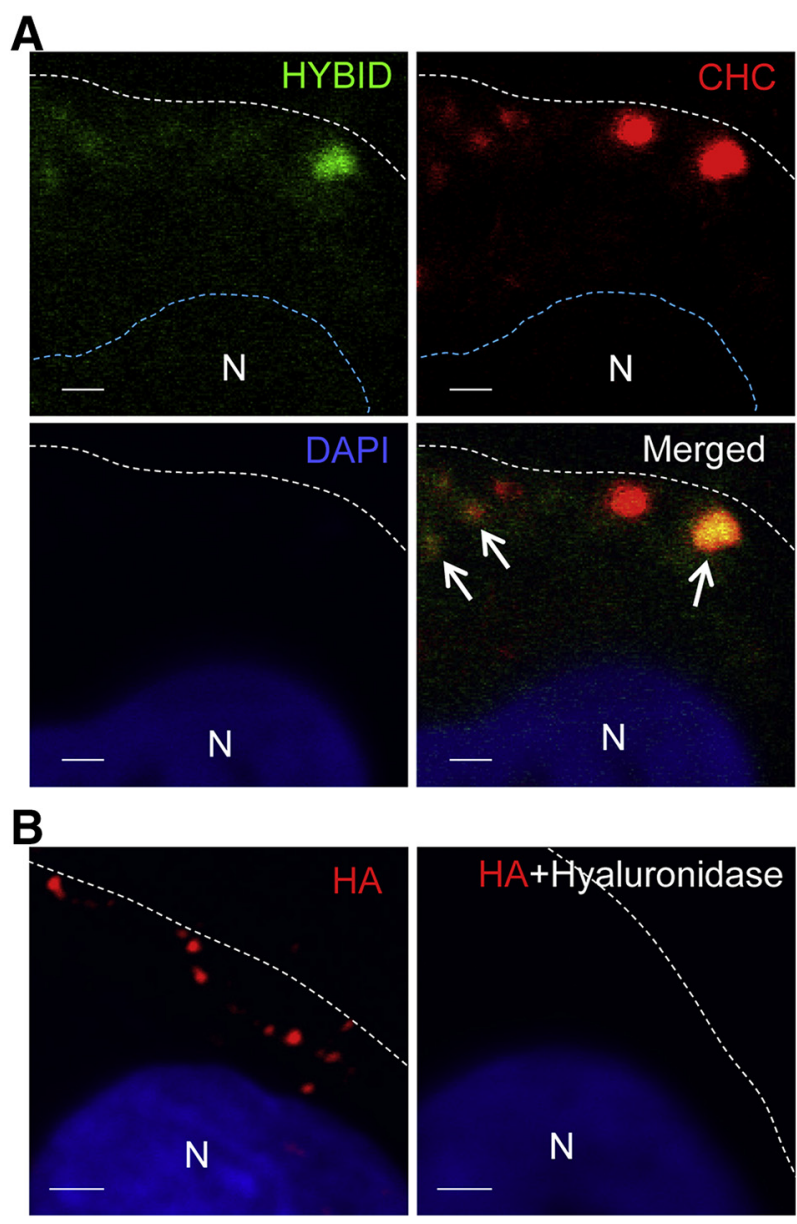

Figure 5 Cellular localization of hyaluronan (HA)-binding protein involved in HA depolymerization (HYBID) and exogenously added high-molecular-weight HA in osteoarthritic (OA) chondrocytes. A: Double immunostaining of HYBID and the clathrin heavy chain $(\mathrm{CHC})$ in $\mathrm{OA}$ chondrocytes. The permeabilized cells were immunostained with antiHYBID antibody conjugated to Alexa Fluor 488 (green) and anti-CHC antibody conjugated to Alexa Fluor 555 (red). The plasma membrane and border of nuclei are depicted by white and blue dashed lines, respectively. Arrows indicate HYBID and CHC double-positive signals. B: Localization of exogenously added HA in OA chondrocytes. The cells were incubated for 1 hour with biotin-labeled high-molecular-weight HA or biotin-labeled highmolecular-weight HA treated with Streptomyces hyaluronidase. After permeabilization, they were followed by reaction with streptavidin conjugated to Alexa Fluor 555. The plasma membrane is shown by the dashed line. Scale bars $=2 \mu \mathrm{m}$. N, nuclei.

chondrocytes, suggesting a potential role of HYAL2 in HA degradation. However, since knockdown of HYAL1 or CD44 resulted in negligible suppression of HA-degrading activity, it seems unlikely that the HYAL2/CD44 and HYAL1 system is a central pathway for HA degradation in OA chondrocytes. On the other hand, OA chondrocytes showed almost complete disappearance of the activity by knockdown of the HYBID expression, co-localization of HYBID with the clathrin heavy chain, and rapid vesicle endocytosis of high-molecular-weight HA. Thus, our data strongly suggest that HYBID is involved in the HA degradation by OA chondrocytes, as we have demonstrated in skin and synovial fibroblasts.
Up-regulation of HYBID in OA cartilage suggests the possibility that the expression by chondrocytes is regulated by factors such as proinflammatory cytokines and growth factors, which are commonly produced in OA joint tissues. Therefore, the effects of eight factors (TNF- $\alpha$, TGF- $\beta_{1}$, IL-1 $\alpha$, histamine, insulin-like growth factor 1 , vascular endothelial growth factor, basic fibroblast growth factor, and prostaglandin $\mathrm{E}_{2}$ ) on HYBID gene expression were studied, and it was found that the expression is promoted only by TNF- $\alpha$ treatment. HYBID was originally identified in human skin fibroblasts as a molecule that is strikingly up-regulated and down-regulated by histamine and TGF- $\beta_{1}$, respectively. ${ }^{9}$ HYBID expression in human skin fibroblasts is down-regulated most efficiently by TGF- $\beta_{1}$ and to some extent by basic fibroblast growth factor, epidermal growth factor, and platelet-derived growth factor $\mathrm{BB} .{ }^{10}$ However, the down-regulation by TGF- $\beta_{1}$ is negligible in arthritic synovial fibroblasts. ${ }^{10}$ According to the different responses to growth factors, regulation of HYBID gene expression was suggested to be cell-type specific. ${ }^{10}$ In the present study, no definite changes in HYBID expression were obtained in OA chondrocytes treated with TGF- $\beta_{1}$, basic fibroblast growth factor, or histamine. In human cancer cell lines such as breast carcinoma cells, hypomethylation of the HYBID (KIAA1199) promoter region is reported to link to HYBID overexpression. $^{34}$ In colon carcinoma cells, hypoxia is known to promote HYBID (CEMIP) expression by binding of hypoxia-inducible factor $2 \alpha$ to the hypoxia response element within the promoter region through increased presence of $\mathrm{H} 3 \mathrm{~K} 4 \mathrm{me} 3$ in the promoter. ${ }^{12}$ Therefore, HYBID expression in OA chondrocytes may also be regulated by the epigenetic mechanism. On the other hand, the promoter region of the HYBID gene contains activator protein 1 and NF- $\kappa \mathrm{B}$ binding sites, and these elements are known to be essential to HYBID gene expression in breast carcinoma cells. ${ }^{34}$ Since activator protein 1 and NF- $\mathrm{BB}$ are the major transcription factors of soluble TNF- $\alpha-\mathrm{TNF}$ receptor 1complex I signaling, ${ }^{35}$ HYBID expression after stimulation with TNF- $\alpha$ in OA chondrocytes may be caused through this pathway, although detailed further studies are needed to clarify the intracellular signaling pathways for the regulation of $H Y B I D$ gene expression.

The present study had potential limitations. HA in articular cartilage is present as the HA-aggrecan network structure, which is formed by the association of aggrecan molecules with HA chain. ${ }^{2,3}$ In the present study, high-molecularweight HA was used for HA digestion by OA chondrocytes. If aggrecan of the HA-aggrecan network in OA cartilage is digested by metalloproteinases such as a disintegrin and metalloproteinase with thrombospondin motifs 4 and 5 before HA degradation by HYBID, the data may fulfill the requirements for HA digestion by HYBID in the cartilage. However, since no information is available on sequential digestion of aggrecan and HA in OA cartilage, how HA in the HA-aggrecan network structure is degraded by HYBID in cartilage tissue deserves to be further investigated. 


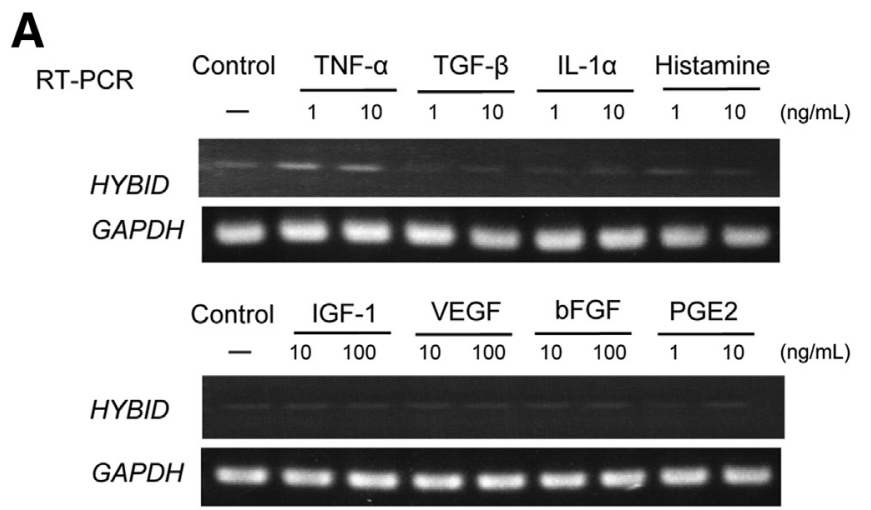

B

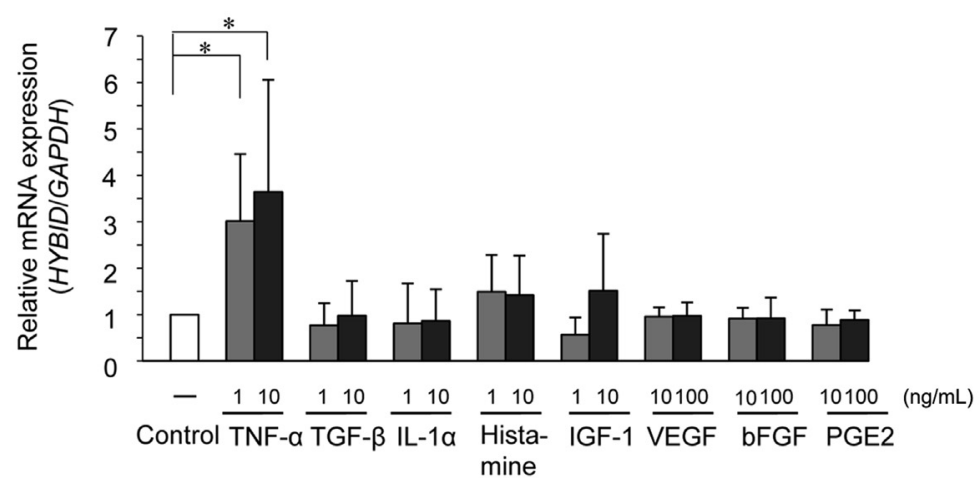

C

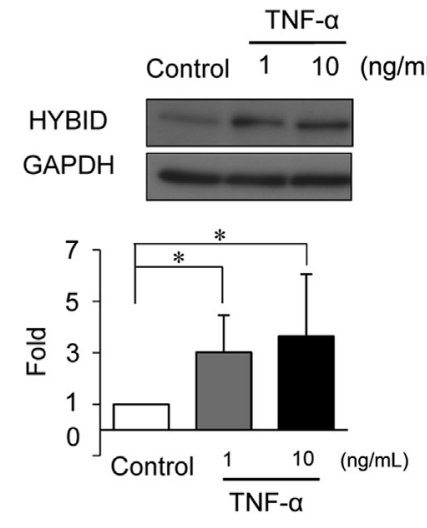

D

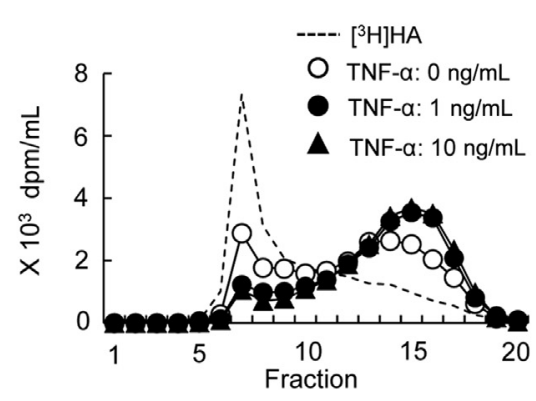

Figure 6 Enhanced expression of hyaluronan (HA)binding protein involved in HA depolymerization (HYBID) by tumor necrosis factor (TNF)- $\alpha$ in osteoarthritic (OA) chondrocytes. A: Effects of TNF- $\alpha$, transforming growth factor (TGF)- $\beta_{1}$, IL-1 $\alpha$, histamine, insulin-like growth factor (IGF)-1, vascular endothelial growth factor (VEGF)-165, basic fibroblast growth factor (bFGF), and prostaglandin $\mathrm{E}_{2}$ (PGE2) on HYBID expression in $\mathrm{OA}$ chondrocytes. $O A$ chondrocytes were treated with these factors or vehicle alone for 24 hours, and HYBID expression was examined by RT-PCR. GAPDH was used as positive control. B: Real-time quantitative PCR analysis of HYBID expression after treatment with the factors. Relative expression of HYBID was determined by normalizing the samples to the expression level of GAPDH transcripts using the $\Delta \Delta C_{\mathrm{T}}$ method. The mean HYBID:GAPDH ratio in control chondrocytes treated with vehicle alone was set at 1 . C: Immunoblot analysis of HYBID expression in OA chondrocytes treated with TNF- $\alpha$. The cells were stimulated without (control) or with TNF- $\alpha$ (1 and $10 \mathrm{ng} / \mathrm{mL}$ ) for 24 hours, and cell lysates were subjected to immunoblot analysis with anti-HYBID antibody. Densitometric quantification was performed in three independent experiments. D: HA-degrading activity in $\mathrm{OA}$ chondrocytes treated with TNF- $\alpha$. OA chondrocytes were stimulated with TNF- $\alpha(0,1$, and $10 \mathrm{ng} / \mathrm{mL}$ ) and cultured with $\left[{ }^{3} \mathrm{H}\right] \mathrm{HA}$ for 48 hours. HAdegrading activity was determined by size-exclusion chromatography. Dashed lines indicate chromatograph for $\left[{ }^{3} \mathrm{H}\right] \mathrm{HA}$ without incubation with chondrocytes; solid lines indicate chromatograph for $\left[{ }^{3} \mathrm{H}\right] \mathrm{HA}$ incubated with chondrocytes treated with TNF- $\alpha$. Data are expressed as means \pm SD (B and $\mathbf{C}$ ). ${ }^{*} P<0.05$.
Nonetheless, HA degradation in the hypertrophic chondrocyte zone is mediated by HYBID expression, ${ }^{24}$ and thus it is plausible that $\mathrm{HA}$ in the HA-aggrecan network may be degraded by the action of HYBID in the OA cartilage tissue. Another question is how the HA-aggrecan network structure remote from chondrocytes is degraded. The recent study showing that HYAL2 is secreted and has weak HAdegrading activity at neutral $\mathrm{pH}^{36}$ suggests the possibility that HYAL2 may be involved in HA degradation in the interterritorial zone. However, further studies are definitely necessary to fully elucidate the catabolic mechanisms of HA in the HA-aggrecan network at the molecular and cellular levels within articular cartilage.
In summary, we have demonstrated that HYBID is overexpressed by chondrocytes in the HA-depleted areas of OA cartilage with a direct correlation with Mankin score, and that HYBID expression is up-regulated by TNF- $\alpha$ in OA chondrocytes. Accumulated lines of evidence have indicated that extracellular matrix-degrading metalloproteinases, including collagenolytic matrix metalloproteinases and aggrecanolytic a disintegrin and metalloproteinase with thrombospondin motifs 4 and 5, play central roles in the degradation of collagens and aggrecan in OA cartilage. ${ }^{2,3,27}$ On the other hand, the present study has provided, to the best of our knowledge, the first data that HYBID is involved in HA degradation in OA cartilage and may be a molecular target in OA patients. Since 
HYBID expression and activity are up-regulated by TNF- $\alpha$, one possible therapeutic strategy may be the application of TNF- $\alpha$ blockade, which has been reported to be effective both for the prevention of TNF- $\alpha$-induced cartilage damage in a preclinical study ${ }^{37}$ and for the treatment of a patient with inflammatory knee OA. ${ }^{38}$ In addition, the development of inhibitors selective to HYBID or molecules to down-regulate expression might be another option, although deliberate and detailed analyses of the function of HYBID in pathophysiologic conditions are still needed for clarification.

\section{Acknowledgments}

We thank Yuko Hashimoto and Chiho Yoshinaga for their technical assistance and Mika Aoki and Tomomi Nakamura, Biological Science Research, Kao Corporation, for their analyses of HA-degradation activity.

\section{References}

1. Nagase H, Kashiwagi M: Aggrecanases and cartilage matrix degradation. Arthritis Res Ther 2003, 5:94-103

2. Okada Y: Proteinases and matrix degradation. Kelley and Firestein's Textbook of Rheumatology, ed 10. Edited by Firestein GS, Budd RC, Gabriel SE, McInnes IB, O’Dell JR. Philadelphia: Elsevier Inc, 2017. pp. $106-125$

3. Fosang AJ, Little CB: Drug insight: aggrecanases as therapeutic targets for osteoarthritis. Nat Clin Pract Rheumatol 2008, 4:420-427

4. Shiomi T, Lemaitre V, D’Armiento J, Okada Y: Matrix metalloproteinases, a disintegrin and metalloproteinases, and a disintegrin and metalloproteinases with thrombospondin motifs in non-neoplastic diseases. Pathol Int 2010, 60:477-496

5. Imai K, Ohta S, Matsumoto T, Fujimoto N, Sato H, Seiki M, Okada Y: Expression of membrane-type 1 matrix metalloproteinase and activation of progelatinase A in human osteoarthritic cartilage. Am J Pathol 1997, 151:245-256

6. Shlopov BV, Lie WR, Mainardi CL, Cole AA, Chubinskaya S, Hasty KA: Osteoarthritic lesions: involvement of three different collagenases. Arthritis Rheum 1997, 40:2065-2074

7. Bau B, Gebhard PM, Haag J, Knorr T, Bartnik E, Aigner T: Relative messenger RNA expression profiling of collagenases and aggrecanases in human articular chondrocytes in vivo and in vitro. Arthritis Rheum 2002, 46:2648-2657

8. Naito S, Shiomi T, Okada A, Kimura T, Chijiiwa M, Fujita Y, Yatabe T, Komiya K, Enomoto H, Fujikawa K, Okada Y: Expression of ADAMTS4 (aggrecanase-1) in human osteoarthritic cartilage. Pathol Int 2007, 57:703-711

9. Yoshida H, Nagaoka A, Kusaka-Kikushima A, Tobiishi M, Kawabata K, Sayo T, Sakai S, Sugiyama Y, Enomoto H, Okada Y, Inoue S: KIAA1199, a deafness gene of unknown function, is a new hyaluronan binding protein involved in hyaluronan depolymerization. Proc Natl Acad Sci U S A 2013, 110:5612-5617

10. Nagaoka A, Yoshida H, Nakamura S, Morikawa T, Kawabata K, Kobayashi M, Sakai S, Takahashi Y, Okada Y, Inoue S: Regulation of hyaluronan (HA) metabolism mediated by HYBID (hyaluronan-binding protein involved in HA depolymerization, KIAA1199) and HA synthases in growth factor-stimulated fibroblasts. J Biol Chem 2015, 290:30910-30923

11. Abe S, Usami S, Nakamura Y: Mutations in the gene encoding KIAA1199 protein, an inner-ear protein expressed in Deiters' cells and the fibrocytes, as the cause of nonsyndromic hearing loss. J Hum Genet 2003, 48:564-570
12. Evensen NA, Li Y, Kuscu C, Liu J, Cathcart J, Banach A, Zhang Q, Li E, Joshi S, Yang J, Denoya PI, Pastorekova S, Zucker S, Shroyer KR, Cao J: Hypoxia promotes colon cancer dissemination through up-regulation of cell migration-inducing protein (CEMIP). Oncotarget 2015, 6:20723-20739

13. Altman R, Asch E, Bloch D, Bole G, Borenstein D, Brandt K, Christy W, Cooke TD, Greenwald R, Hochberg M: Development of criteria for the classification and reporting of osteoarthritis. Classification of osteoarthritis of the knee. Diagnostic and Therapeutic Criteria Committee of the American Rheumatism Association. Arthritis Rheum 1986, 29:1039-1049

14. Altman R, Alarcon G, Appelrouth D, Bloch D, Borenstein D, Brandt K, Brown C, Cooke TD, Daniel W, Feldman D: The American College of Rheumatology criteria for the classification and reporting of osteoarthritis of the hip. Arthritis Rheum 1991, 34:505-514

15. Mankin HJ, Dorfman H, Lippiello L, Zarins A: Biochemical and metabolic abnormalities in articular cartilage from osteo-arthritic human hips. II. Correlation of morphology with biochemical and metabolic data. J Bone Joint Surg Am 1971, 53:523-537

16. Fujita Y, Shiomi T, Yanagimoto S, Matsumoto H, Toyama Y, Okada Y: Tetraspanin CD151 is expressed in osteoarthritic cartilage and is involved in pericellular activation of pro-matrix metalloproteinase 7 in osteoarthritic chondrocytes. Arthritis Rheum 2006, $54: 3233-3243$

17. Meyer LJ, Stern R: Age-dependent changes of hyaluronan in human skin. J Invest Dermatol 1994, 102:385-389

18. Chijiiwa M, Mochizuki S, Kimura T, Abe H, Tanaka Y, Fujii Y, Shimizu H, Enomoto H, Toyama Y, Okada Y: CCN1 (Cyr61) is overexpressed in human osteoarthritic cartilage and inhibits ADAMTS-4 (aggrecanase 1) activity. Arthritis Rheumatol 2015, 67: $1557-1567$

19. Enomoto H, Inoki I, Komiya K, Shiomi T, Ikeda E, Obata K, Matsumoto H, Toyama Y, Okada Y: Vascular endothelial growth factor isoforms and their receptors are expressed in human osteoarthritic cartilage. Am J Pathol 2003, 162:171-181

20. Flannery CR, Little CB, Hughes CE, Caterson B: Expression and activity of articular cartilage hyaluronidases. Biochem Biophys Res Commun 1998, 251:824-829

21. Chow G, Knudson CB, Knudson W: Expression and cellular localization of human hyaluronidase-2 in articular chondrocytes and cultured cell lines. Osteoarthritis Cartilage 2006, 14:849-858

22. Salter DM, Godolphin JL, Gourlay MS, Lawson MF, Hughes DE, Dunne E: Analysis of human articular chondrocyte CD44 isoform expression and function in health and disease. J Pathol 1996, 179: 396-402

23. Michishita E, Garces G, Barrett JC, Horikawa I: Upregulation of the KIAA1199 gene is associated with cellular mortality. Cancer Lett 2006, 239:71-77

24. Shimoda M, Yoshida H, Mizuno S, Hirozane T, Horiuchi K, Yoshino Y, Hara H, Kanai Y, Inoue S, Ishijima M, Okada Y: Hyaluronan-binding protein involved in hyaluronan depolymerization controls endochondral ossification through hyaluronan metabolism. Am J Pathol 2017, 187:1162-1176

25. van der Kraan PM, van den Berg WB: Chondrocyte hypertrophy and osteoarthritis: role in initiation and progression of cartilage degeneration? Osteoarthritis Cartilage 2012, 20:223-232

26. Mwale F, Billinghurst C, Wu W, Alini M, Webber C, Reiner A, Ionescu M, Poole J, Poole AR: Selective assembly and remodelling of collagens II and IX associated with expression of the chondrocyte hypertrophic phenotype. Dev Dyn 2000, 218:648-662

27. Pratta MA, Yao W, Decicco C, Tortorella MD, Liu RQ, Copeland RA, Magolda R, Newton RC, Trzaskos JM, Arner EC: Aggrecan protects cartilage collagen from proteolytic cleavage. J Biol Chem 2003, 278: 45539-45545

28. Dicker KT, Gurski LA, Pradhan-Bhatt S, Witt RL, Farach-Carson MC, Jia X: Hyaluronan: a simple polysaccharide with diverse biological functions. Acta Biomater 2014, 10:1558-1570 
29. Hascall VC, Wang A, Tammi M, Oikari S, Tammi R, Passi A, Vigetti D, Hanson RW, Hart GW: The dynamic metabolism of hyaluronan regulates the cytosolic concentration of UDP-GlcNAc. Matrix Biol 2014, 35:14-17

30. Bourguignon LY, Singleton PA, Diedrich F, Stern R, Gilad E: CD44 interaction with $\mathrm{Na}^{+}-\mathrm{H}^{+}$exchanger (NHE1) creates acidic microenvironments leading to hyaluronidase-2 and cathepsin B activation and breast tumor cell invasion. J Biol Chem 2004, 279: 26991-27007

31. Harada H, Takahashi M: CD44-dependent intracellular and extracellular catabolism of hyaluronic acid by hyaluronidase-1 and -2. J Biol Chem 2007, 282:5597-5607

32. Csoka AB, Scherer SW, Stern R: Expression analysis of six paralogous human hyaluronidase genes clustered on chromosomes 3p21 and 7q31. Genomics 1999, 60:356-361

33. Triggs-Raine B, Salo TJ, Zhang H, Wicklow BA, Natowicz MR: Mutations in HYAL1, a member of a tandemly distributed multigene family encoding disparate hyaluronidase activities, cause a newly described lysosomal disorder, mucopolysaccharidosis IX. Proc Natl Acad Sci U S A 1999, 96:6296-6300

34. Kuscu C, Evensen N, Kim D, Hu YJ, Zucker S, Cao J: Transcriptional and epigenetic regulation of KIAA1199 gene expression in human breast cancer. PLoS One 2012, 7:e44661

35. Kalliolias GD, Ivashkiv LB: TNF biology, pathogenic mechanisms and emerging therapeutic strategies. Nat Rev Rheumatol 2016, 12:49-62

36. Hida D, Danielson BT, Knudson CB, Knudson W: CD44 knock-down in bovine and human chondrocytes results in release of bound HYAL2. Matrix Biol 2015, 48:42-54

37. Urech DM, Feige U, Ewert S, Schlosser V, Ottiger M, Polzer K, Schett G, Lichtlen P: Anti-inflammatory and cartilage-protecting effects of an intra-articularly injected anti-TNF\{alpha\} single-chain Fv antibody (ESBA105) designed for local therapeutic use. Ann Rheum Dis 2010, 69:443-449

38. Grunke M, Schulze-Koops H: Successful treatment of inflammatory knee osteoarthritis with tumour necrosis factor blockade. Ann Rheum Dis 2006, 65:555-556 